\title{
FRAGILIDAD Y DEBILIDAD COMO ELEMENTOS FUNDAMENTALES DEL ESTEREOTIPO TRADICIONAL FEMENINO
}

\author{
ESPERANZA BOSCH FIOL Y VICTORIA A. FERRER PÉREZ \\ Universidad de las Islas Baleares
}

Las autoras que firmamos este artículo llevamos ya más de diez años interesadas en el estudio de la construcción y transmisión del estereotipo femenino. Durante todo este tiempo hemos aprendido muchas cosas que nos ayudan a entender mejor la realidad en la que vivimos. Hemos analizado muchos textos, tanto actuales como del pasado, y otros tantos estudios realizados desde diferentes disciplinas. También hemos entrevistado a muchas mujeres, hemos hablado con ellas de sus relaciones con los hombres, de la violencia de género, de la relación con sus propios cuerpos, de sus vivencias y de sus temores. Por otra parte, hemos recabado la opinión de los hombres sobre sus compañeras sentimentales, sobre sus relaciones amorosas. Toda esta información nos ha permitido constatar algo que, por otra parte, ya se venía denunciando en muy diversos foros: la persistencia de muchos mitos y falsas creencias sobre la feminidad y la masculinidad y el fuerte contenido misógino que los alimenta. A pesar de que en nuestro mundo occidental todos estos prejuicios puedan manifestarse de manera más sutil (aunque no siempre), su persistencia dificulta enormemente la vida y aspiraciones de la mitad de la población, y están en la base de las situaciones injustas que frenan, si no impiden, la igualdad de oportunidades y el principio, constitucionalmente definido, de no discriminación en función del sexo.

En este artículo proponemos una reflexión sobre algunos de estos componentes misóginos del estereotipo femenino y la modalidad que éste adopta en la actualidad.

Creemos firmemente que nadie puede dudar de los avances fundamentales que se han conseguido en las últimas décadas en la lucha por la igualdad gracias, muy especialmente, al movimiento feminista. Sin embargo, el patriarcado tiene unas profundas raíces enquistadas en las mentalidades, las costumbres y las normas sociales. Acabar con ellas implica un enorme esfuerzo, tanto de activismo como de análisis y cuestionamiento de la realidad, especialmente de

Feminismo/s, 2, diciembre 2003, pp. 139-152 
aquella más cercana a nuestra vida cotidiana que, por tanto, es también la que más nos puede condicionar.

\section{PERFILES MISÓGINOS EN LA CONSTRUCCIÓN DEL ESTEREOTIPO TRADICIONAL FEMENINO}

En The Fontana Dictionary of Modern Thought, Stallybrass definió el estereotipo como:

«una imagen mental generalmente muy simplificada de alguna categoría de personas, institución o acontecimiento que es compartida en sus características esenciales por un gran número de personas. Los estereotipos van frecuentemente, aunque no necesariamente, acompañados de prejuicios, es decir, de una predisposición favorable o desfavorable hacia cualquier miembro de esa categoría en cuestión» ${ }^{1}$.

Así pues, vemos cómo el estereotipo es algo subjetivo que descansa en ideas preconcebidas, condiciona las opiniones de las personas y dirige las expectativas de los miembros del grupo social, es decir, determina qué es lo que esperamos unos de otros y cómo nos vemos unos a otros.

Un subtipo de los estereotipos sociales en general son los estereotipos de género. Podríamos definir éstos como creencias o pensamientos que las personas tenemos sobre cuáles son y cuáles deberían ser los atributos personales de hombres y mujeres. Autores/as como Ashmore ${ }^{2}$, Kay Deaux y Laurie L. Lewis ${ }^{3}$ o Janet T. Spence 4 muestran que están formados por varias dimensiones que, aunque relacionadas entre sí, actúan con independencia. Así, los estereotipos de género incluirían las creencias populares sobre aspectos como los rasgos de personalidad, las conductas correspondientes al rol, las ocupaciones laborales o la apariencia física que se consideran características de los hombres y de las mujeres y que diferencian a los unos de las otras.

En relación concreta al estereotipo femenino, vemos cómo éste se ha ido nutriendo, a lo largo de los siglos, de todo un enorme listado de rasgos o características supuestamente propias de las mujeres, pero con un eje central muy claro: la inferioridad de éstas en relación a los hombres. Esta supuesta inferioridad se ha ido sustentando en tres argumentos básicos: la inferioridad moral, la intelectual y la biológica ${ }^{5}$. Es decir, se ha dado por sentado que las mujeres,

1. Staliybrass, O.: "Stereotype», en A. Bullock y O. Stallybrass (dirs.): The Fontana Dictionary of a Modern Tough, Londres, Fontana/Collins, 1977, p. 490.

2. Ashmore, R. D.: "Sex stereotypes and impact personality theory", en D.L. Hamilton (ed.): Cognitive processes in stereotipyng and intergroup behavior, Hillsdale, Erlbaum, 1981.

3. Deaux, Kay y Lewis, Laurie L.: "Components of gender stereotypes", Psychological Documents, 13 (1983), p. 25.

Deaux, Kay y Lewis, Laurie L.: "The structure of gender stereotypes. Interrelations ships among components of gender label», Journal of Personality and Social Psychology, 45 (1984), pp. 991-1004.

4. SenCE, Janet T.: "Gender identity and its implications for the concepts of masculinity and feminity", en T.B. Sonderegger (ed.): Nebraska Symposium Motivation: Psychology and Gender, Lincoln, Univ. Nebraska Press, vol. 32, 1985, pp. 59-96.

5. Bosch, Esperanza, Ferrer, Victoria A. y GILI, Margarita: Historia de la misoginia, Barcelona, Antrophos - UIB, 1999. 
por el hecho de serlo, son más laxas desde el punto de vista moral, siguiendo el modelo de Eva o Pandora (prototipos de mujeres desobedientes y curiosas cuya flaqueza moral trajo grandes perjuicios para la humanidad); son menos inteligentes que los hombres, menos dotadas de capacidad racional, aunque eso sí, más intuitivas y emotivas; y, desde el punto de vista físico y biológico, más frágiles y enfermizas y dependientes de los caprichos hormonales: el llamado el sexo débil.

Tanto la cultura oficial como la popular han alimentado, y alimentan todavía, estos prejuicios utilizándolos como justificación para el mantenimiento de una determinada situación de privilegio masculino. Las mujeres, tal y como el estereotipo las retrata, serán consideradas como «naturalmente» destinadas a la vida privada, a la maternidad y al cuidado de la familia, y poco interesadas por cualquier otra actividad, especialmente aquellas vinculadas al ámbito público.

Este estado de cosas cristalizará en el contexto de la familia patriarcal y, por extensión, de la sociedad patriarcal, donde la autoridad masculina no se cuestiona, y el uso de la violencia para corregir las desviaciones o desmanes femeninos se legitima. Diversos ámbitos del conocimiento acudirán en ayuda del patriarcado. En el caso de la psicología, una rama de la misma, la psicología diferencial, dio por sentadas estas asimetrías, considerándolas como consustanciales a la "esencia femenina».

Vamos a repasar a continuación algunos de los rasgos determinantes que durante mucho tiempo han definido cómo deben ser y comportarse un hombre «masculino» y una mujer "femenina». El trabajo de Williams y Bennett ${ }^{6}$ nos ofrece un buen ejemplo de ello, mostrando las características que más frecuentemente aparecen en uno y otro estereotipos de género, y que se presentan en el cuadro siguiente :

\begin{tabular}{|ll|ll|}
\hline \multicolumn{2}{|c|}{ MUJERES } & \multicolumn{2}{c|}{ HOMBRES } \\
Afectada & Gentil & Agresivo & Excitable \\
Atractiva & Habladora & Ambicioso & Fuerte \\
Complaciente & Llorona & Asertivo & Hábil \\
Coqueta & Machacona & Austero & Independiente \\
De corazón blando & Remilgada & Auto-confiado & Lógico \\
Débil & Sensible & Autocrático & Masculino \\
Dependiente & Sentimental & Aventurero & No emotivo \\
Dócil & Sofisticada & Con coraje & Racional \\
Emocional & Soñadora & Cruel & Realista \\
Encantadora & Sumisa & Desordenado & Resistente \\
Estirada & Voluble & Digno de confianza & Robusto \\
Excitable & & Dominante & Seguro \\
Femenina & & Emprendedor & Severo \\
Frívola & Estable & \\
& & & \\
\hline
\end{tabular}

6. Wlliams, J. E. y BenetT, S. M.: "The definition of sex stereotypes via the Adjective Check List», Sex Roles, 1 (1975), pp. 327-337. 
Por su parte, Sandra Bem creó un cuestionario, el «Bem Sex Role Inventory», que responde a una nueva concepción de la masculinidad y la feminidad ${ }^{7}$. La idea central es considerar que éstas son dos dimensiones independientes, de las que una abarca desde el "nada femenino hasta el muy femenino" y la otra desde el «nada masculino al muy masculino». Estas dos dimensiones no se entienden como necesariamente excluyentes, pudiendo ocurrir que una persona tenga al mismo tiempo características psicológicas masculinas y femeninas (siendo, por tanto, andrógina). Las características típicas de una y otra dimensión que se recogen en el citado cuestionario son las siguientes:

\begin{tabular}{|l|l|}
\hline MASCULINIDAD & FEMINIDAD \\
Actúa como líder & Acogedor/a \\
Agresivo/a & Actúa con simpatía \\
Ambicioso/a & Afectuoso/a \\
Analítico/a & Alegre \\
Asertivo/a & Compasivo/a \\
Atlético/a & Comprensivo/a \\
Autosuficiente & Condescendiente \\
Competitivo/a & Cortés \\
Con capacidad de liderazgo & Crédulo/a \\
Confiado/a en sí mismo/a & Femenino/a \\
De personalidad fuerte & Habla con suavidad \\
Decide con facilidad & Impaciente por aliviar sentimientos de daño \\
Defensor/a de sus propias creencias & Infantil \\
Dispuesto/a a arriesgarse & Le gustan los/as niños/as \\
Dispuesto/a a tomar partido & Leal \\
Dominante & No es malhablado/a \\
Independiente & No se deja adular \\
Individualista & Sensible ante las necesidades de los demás \\
Masculino/a & Tierno/a \\
Vigoroso/a & Tímido/a \\
\hline
\end{tabular}

Así pues, aunque la concepción de Sandra Bem sea más novedosa en algunos aspectos (por ejemplo, al sugerir que una persona puede tener simultáneamente características de las consideradas típicamente masculinas y de las consideradas típicamente femeninas, o al considerar que esa circunstancia dota a la persona de una mayor capacidad de adaptación al medio), en realidad no rompe la idea de que existen unas características masculinas tradicionales y otras características femeninas tradicionales.

7. BeM, Sandra: «The measurement of psychological androgyny», Journal of Consulting and Clinical Psychology, 42 (1974), pp. 155-162. 
A continuación, y de una manera muy simple por necesidades de espacio, compararemos algunos de los rasgos considerados como típicamente masculinos y femeninos con aquellos síntomas más frecuentes en los cuadros clínicos depresivos:

DEPRESIÓN
Dependencia
Pasividad
Falta de firmeza o asertividad
Gran necesidad de apoyo afectivo
Baja autoestima e indefensión
Incompetencia
DEPRESIÓN
Dependencia
Pasividad
Falta de firmeza o asertividad
Gran necesidad de apoyo afectivo
Baja autoestima e indefensión
Incompetencia

\section{FEMINIDAD}

Dependencia

Pasividad

Falta de firmeza o asertividad

Gran necesidad de apoyo afectivo

Como efecto, se desarrolla baja autoestima

e indefensión

Incompetencia

\author{
MASCULINIDAD \\ Autonomía \\ Actividad \\ Asertividad y agresividad \\ Capacidad de aceptar riesgos y tomar \\ decisiones \\ Como efecto, se desarrolla alta autoestima \\ y seguridad \\ Competencia
}

La conclusión salta a la vista: muchos de los rasgos atribuidos al modelo de feminidad se relacionan con una sintomatología depresiva. Esto significa que socializar a las niñas enseñándolas a comportarse «de manera típicamente femenina", conlleva, de alguna manera, enseñarles también a infravalorarse, a sentirse dependientes e inseguras, $y$, por tanto, a convertirse en más vulnerables ante la depresión.

En este sentido, ya la psicóloga Gloria Poal nos recordaba que haber nacido varón tiene ventajas y desventajas. Las primeras se pueden resumir así: a) ese sexo inspira orgullo y prestigio; b) se le presuponen virtudes; c) disponen de más libertad; d) se les permiten más transgresiones; y e) se les estimula más hacia el éxito.

Entre las desventajas, esta autora señala: a) cargan con múltiples expectativas (exitosos, valientes, etc.); b) se les exigen esfuerzo y logros; c) se les prohíbe expresar miedo e inseguridad; d) se les da poco apoyo afectivo, se les conforta poco; y e) han de reprimir la expresión de los afectos.

Sin embargo, según señala la autora, haber nacido mujer no tiene ventajas propiamente dichas puesto que es el sexo menos valorado. Por el contrario, tiene asignadas, como finalidad de su existencia, la mayor parte de tareas reproductivas en sentido amplio 8 .

Lo que sí tiene el nacer mujer son múltiples desventajas, entre las que se encuentran, precisamente, las relacionadas con el estereotipo de fragilidad que

8. POAL, Gloria: Entrar, quedarse, avanzar, Madrid, Siglo XXI, 1993. 
«justifica» que le limiten sus movimientos y su radio de acción, lo cual acaba generando inseguridad, miedo y baja autoestima9.

\section{FRAGILIDAD Y DEBILIDAD: JUSTIFICACIONES PARA LA DEPENDENCIA}

Tras este breve análisis de las características que se han considerado parte del estereotipo tradicional femenino, vamos ahora a ampliar un poco más el tema de la fragilidad femenina como rasgo atribuido de forma natural a las mujeres, y que justifica su subordinación.

Pensadores de la talla de Rousseau afirmaban algo que en el siglo XIX se tuvo muy claro. Decía el ilustre pensador que la distinción jerárquica de poder, de dignidad, de autoridad, de saber entre varones y mujeres no era producto de un esfuerzo para que la distinción existiera, era, por el contrario, natural. Y lo natural debía seguir existiendo sin ponerle ninguna cortapisa. Al parecer este autor no veía contradicción alguna en ser demócrata para los varones y naturalista para las mujeres.

Como nos recuerda Amelia Valcárcel, los románticos, a la vez que construyeron la ficción de la mujer ideal, dejaron a las mujeres reales sin derechos ${ }^{10}$.

Inmediatamente la ciencia se lanzó en defensa de todos estos prejuicios, y fueron surgiendo un gran número de teorías, consideradas científicas en su momento, que pretendieron demostrar la inferioridad de las mujeres en sus diversas vertientes y su destino natural al cuidado de la familia.

Algunos ejemplos de lo anterior los encontraríamos en la frenología capitaneada por Gall, que defendía la relación entre el menor tamaño del cerebro femenino y su menor capacidad intelectual. O Moebius cuya obra titulada La inferioridad mental de las mujeres ya indicaba claramente por dónde iban los tiros, centrando también sus argumentos, como hicieran los frenólogos, en el tamaño cerebral. Más ejemplos: Kormiloff y Melassez creyeron poder demostrar que la sangre de las mujeres contenía menos corpúsculos rojos, menos hemoglobina y más agua que la de los varones.

Otros insignes médicos como Quételet, Wisberg, Andral y Scharling, defendían la menor capacidad pulmonar, esquelética, fonadora, etc., y de todo ello derivaban la debilidad y situación de enferma crónica que podía atribuirse a las mujeres. Y el biólogo y sociólogo británico Herbert Spencer afirmó que la actividad intelectual era incompatible con la procreación por lo que las mujeres no debían estudiar y, además, su mente se degradaba a medida que engendraban hijos.

Siglos atrás Juan Huarte de San Juan, médico renacentista, había defendido la misma tesis aplicando las teorías humorales propias de la época.

La idea central dominante era que la fisiología femenina (embarazos, menstruaciones, partos, menopausia, etc.) situaba a las mujeres en un estado permanente de enfermedad física y de un cierto trastorno de sus facultades mentales.

9. JAYMe, María y SAU, Victoria: Psicología diferencial del sexo y género, Barcelona, Icaria, 1996. 10. VAlCÁRCel, Amelia: La política de las mujeres, Valencia, Cátedra, 1997. 
Todo ello vendría a justificar su subordinación al varón, al que necesitaría como protector y guía, y su dedicación al servicio de la familia, al mundo privado.

Como nos recuerdan diversas autoras, Pilar Ballarín entre ellas, la escolarización obligatoria de las niñas, que se generalizó en el XIX (en España a partir de la ley Moyano de 1857, que obligaba al mantenimiento de una escuela de niñas cada 500 habitantes), servirá para la difusión de estas creencias, legitimándolas desde la institución escolar ${ }^{11}$. La escuela mantendrá durante mucho tiempo currícula diferenciados para niños y niñas y los valores del modelo clásico (castidad, compostura, discreción, frugalidad, ...) impregnaron los contenidos de los aprendizajes destinados a las chicas.

Así, la educación femenina tenía una vertiente de "adorno» y otra de "utilidad doméstica» en la que se enseñaba a las mujeres a realizar las denominadas "tareas propias de su sexo». Todo ello era considerado como consustancial al hecho de haber nacido mujer.

Vamos a ver a continuación un breve texto que puede, perfectamente, ilustrar lo dicho, y que añade un nuevo factor: el sentimiento de culpabilidad que se intenta inculcar a las mujeres que no saben, o, peor todavía, no quieren, cumplir con sus supuestas obligaciones.

«No hemos de olvidar que la mujer está destinada por Dios para desempeñar la más alta misión sobre la tierra, la de ser madre y (...) tiene en sus manos la formación de sus hijos que han de ser los que formen la sociedad.

¿Creéis vosotras, mujeres que esto leáis, que si en España todas, absolutamente todas las madres, hubieran cumplido exactamente los deberes que Dios les ha enseñado, hubiera sido posible la tragedia que estamos padeciendo?»»12

Ciencia, ideología y religión se dan al mano sin ningún tipo de pudor a la hora de justificar la situación de dependencia femenina y su sumisión a la autoridad masculina. Es sin duda cierto que el ideal de mujer romántica era el de la esposa espiritual y frágil, amorosa esposa y madre, etérea en una nube de tules y sedas, pero también lo es la emergencia de otro tipo de mujeres, herederas de la ilustradas, aquellas que reclamaban sus derechos de ciudadanas. Nos referimos a las primera sufragistas.

El sufragismo, movimiento de defensa del derecho al voto femenino nacido en el seno del liberalismo, debió oponerse frontalmente al naturalismo. Había que denunciar la trampa mortal que resulta para las mujeres, es decir, para la mitad de la humanidad, la atribución a los factores biológico-naturales de la esencia de la feminidad, la consideración de que la situación de dependencia y control sobre la vida de millones de mujeres en el mundo debía ser entendida como consecuencia de la misma naturaleza femenina.

11. Ballarín, Pilar: "La construcción de un modelo educativo de 'utilidad doméstica'», en Georges Duby y Michele Perrot: Historia de las Mujeres, Madrid, Taurus, 1993, vol. 4, pp. 598-611.

12. La Mujer de Acción Católica, n 13, junio de 1938, cit. por Escartín, Joana M. y Serrano, Aina: La dona a la Mallorca contemporànea, Palma, Documenta Balear, 1997, p 55. 
Como era de esperar, el peso de la descalificación y el rechazo cayó sobre ellas, y fueron tildadas de "frustradas», "amargadas», «resentidas», y ridiculizadas llamándolas "hombrunas», "feas», "histéricas», «lesbianas». El fantasmagórico miedo masculino se desató de nuevo. Mujeres organizadas que replicaban, defendían públicamente sus derechos, y, aunque con métodos pacíficos, reclamaban su espacio en el mundo, eran todo lo contrario del modelo domesticado de mujer «femenina y frágil», eran, por tanto, un peligro.

Como ya señaló la escritora prematuramente fallecida Montserrat Roig en un ensayo sobre el tema:

"Todavía en nuestros tiempos la palabra feminismo da miedo. Todavía ser 'feminista' significa, para algunas mujeres, distanciarse de los hombres, ser una mujer distinta, agresiva, amenazadora de la paz y de la convivencia.. ${ }^{13}$

Es evidente que las críticas al feminismo y a las feministas son hoy en día, al menos en nuestro contexto cultural, más sutiles que en tiempos pasados, pero sin duda existen, y pretenden mantener una visión distorsionada de este movimiento y un descrédito de sus objetivos. Desde esta perspectiva, podríamos entender mejor los resultados de diversos estudios más o menos recientes ${ }^{14}$, realizados tanto en España como fuera de ella, en los que menos de una tercera parte de las mujeres entrevistadas se consideran a sí mismas feministas. Sin embargo, cuando se les pregunta su opinión sobre temas que tienen que ver con la igualdad de oportunidades no hay diferencias significativas entre quienes se consideran feministas y quienes no se consideran como tales, y unas y otras están básicamente de acuerdo con esa igualdad. Lo que rechazan, y quizás temen, muchas de ellas es el término.

Pero, con todo, el movimiento feminista ha seguido adelante. Con el tiempo el derecho al voto se fue consiguiendo (aunque llegó en fecha muy tardía en algunos países), pero con él no llegaron todas las transformaciones sociales con las que las mujeres soñaban. Por el contrario, lo que emergió como respuesta a tanta aspiración de autonomía fue la llamada mística de la feminidad. Este concepto fue desarrollado por Betty Friedan para describir el conjunto de maniobras ideológicas ejercidas para mantener a las mujeres dentro del hogar ${ }^{15}$.

Por otra parte, y aun cuando se pudiera ejercer el derecho al voto, la realidad mostraba que la participación política de las mujeres era mínima, así como

13. Rorg, Montserrat: Mujeres en busca de un nuevo humanismo, Barcelona, Salvat-Temas clave, 1981, p. 4.

14. Folguera, Pilar: «De la transición política a la democracia. La evolución del feminismo en España durante el período 1975-1988", en Pilar Folguera (comp.): El feminismo en España: Dos siglos de historia, Madrid, Ed. Pablo Iglesias, 1988, pp. 111-131. IDES: Las españolas ante la política, Madrid, Instituto de la Mujer, 1988. La mujer en España. Situación social, Madrid, Instituto de la Mujer, 1990. Hyde, Janet S.: Psicología de la mujer. La otra mitad de la experiencia humana, Madrid, Morata, 1995. Ferrer, Victoria A. y Bosch, Esperanza: «Ser feminista en la España de los 90: Un análisis desde la psicología", Psykhe (Revista de la Escuela de Psicología. Facultad de Ciencias Sociales. Pontificia. Universidad Católica de Chile), 7(2) (1998), pp. 25-32.

15. Friedan, Betty: La mística femenina, Madrid, Ediciones Júcar, 1974. 
también lo era su presencia en cargos de responsabilidad en las diferentes profesiones.

Así las cosas, en los años sesenta, las mujeres, especialmente desde la izquierda, se dan cuenta de que, en las organizaciones políticas en las que participan y en los grupos u organizaciones donde llevan a cabo su militancia ciudadana, están ocupando los mismo lugares subordinados que mantienen en las instituciones familiares o en otros ámbitos de la sociedad civil. Por tanto deciden que hay que alcanzar la absoluta igualdad jurídica. El gran lema es: "Lo personal es político». En palabras de Amelia Valcárcel lo que se viene a denunciar es que no se puede dividir la esfera de acción de un ser humano mujer en cosas que son políticas y otras que son personales ${ }^{16}$. Para las mujeres su vida personal es tal y se desarrolla en tales condiciones que tienen que hacer de ella política, tienen que darse cuenta de que las trabas que tienen son políticas.

Va pasando el tiempo. En los años ochenta el predominio masculino continúa en cualquier rama importante de actividad, política o profesional, sin importar que existan mujeres cualificadas para llevar a cabo cualquier tarea. A ellas se les está vedando de una manera no explícita el ejercicio de actividades significativas que comporten poder. Es evidente la existencia de un techo de cristal, de unas limitaciones no escritas (aunque algunas veces también) que marcan la frontera a partir de la cual el acceso de las mujeres resulta casi imposible. Surgen entonces las declaraciones a favor de la llamada "democracia paritaria», concepto que cristaliza a partir de la «Declaración de Atenas» de $1992^{17}$, y con ellas la búsqueda de compromisos explícitos por parte de los diversos gobiernos para que se arbitren medidas activas para asegurar la presencia de no menos del $40 \%$ ni más del $60 \%$ de hombres y mujeres en los puestos públicos de toma de decisión, y a su vez incentivar el reparto equitativo de las tareas y responsabilidades domésticas y familiares.

Sin embargo, algo más está pasando. Gracias a todos los esfuerzos descritos, en las últimas décadas las mujeres están filtrándose en la estructura del poder, con muchas dificultades, es cierto, pero queremos creer que de una manera imparable, al menos en el mundo occidental. Pero al mismo tiempo los trastornos de alimentación han ido aumentado de forma alarmante, y la cirugía estética se ha convertido en una de las especialidades médicas de más brillante ascenso. La mujer triunfadora, según los cánones actuales, tiene que ser también bella, y la belleza se relaciona directamente con la juventud y con un cuerpo delgado, muy delgado. Parece como si el peaje que las mujeres en su lucha por la emancipación tuvieran que pagar estuviera relacionado con el maltrato a su propio

16. Valcárcel, Amelia: Op. cit.

17. A petición de la Comisión de la Comunidad Europea, tuvo lugar el 3 de noviembre de 1992 en Atenas la primera cumbre europea "Mujeres al poder», que reunió a mujeres ministras o ex-ministras, denunció el déficit democrático existente, proclamó la necesidad de conseguir un reparto equilibrado de los poderes públicos y políticos entre hombres y mujeres y adoptó la denominada «Declaración de Atenas». 
cuerpo y con el mantenimiento de una batalla, imposible de ganar, contra el paso del tiempo.

Las llamadas revistas femeninas mandan mensajes continuos sobre cómo mantener la juventud y belleza, los trucos más eficaces, los productos imprescindibles, las dietas más rápidas, etc., etc. Las industrias de cosmética y dietética son de las más solventes del mercado. Todo ello envuelve, una vez más, el concepto de feminidad. Juventud y delgadez son características fácilmente relacionables con la fragilidad y la dependencia.

La mujer-mujer deberá dedicar buena parte de su presupuesto y de su tiempo para conseguir, o al menos intentar, que su cuerpo se ajuste a los modelos estéticos dominantes, independientemente de cuales sean sus cualidades intelectuales o sus responsabilidades profesionales. Deberá vestirse a la moda, sacrificar comodidad por estética, considerando ambas cosas como prácticamente incompatibles.

\section{EL CUERPO FEMENINO. LA FEMINIDAD COMO ESCLAVITUD}

«El mito de la belleza se basa en esto: la cualidad llamada belleza tiene existencia universal y objetiva. Las mujeres deben aspirar a personificarla y los hombres deben aspirar a poseer mujeres que la personifiquen. Es un imperativo para las mujeres pero no para los hombres, y es necesaria y natural porque es biológica, sexual y evolutiva. Los hombres fuertes luchan por poseer mujeres bellas, y las mujeres bellas tienen mayor éxito reproductivo.» ${ }^{18}$

Las maniobras ejercidas desde el poder patriarcal para el sometimiento de las mujeres van cambiando según las épocas, pero con un denominador común: atacar la autoestima femenina. Nada más útil para mantener el sometimiento de un individuo o grupo que alimentar la creencia en su falta de capacidad, en su falta de atractivo, en su inferioridad. Una persona insegura es una persona más fácilmente controlable. Puesto que muchos de los atributos relacionados con la feminidad se relacionan con aspectos externos, corporales, uno de los flancos típicos para dañar la autoimagen de las mujeres son las alusiones estéticas. De ello no se escapa nadie, ni las mujeres del mundo del arte o la cultura, ni las que ocupan cargos de responsabilidad pública, ni la amas de casa, ni las solteras, ni las casadas. Al ojo escrutador masculino, y al femenino que se identifica con él, no se le escapa nada ni nadie. Alusiones a los supuestos kilos de más, a la celulitis, a las arrugas, a la flacidez o al envejecimiento son permanentes en el discurso sobre las mujeres en la actualidad. La autoestima es la gran víctima.

Definir el concepto de autoestima no es tarea fácil. Ya en 1890 el psicólogo norteamericano William James trató de explicarlo considerando que la autoestima de una persona era igual a su éxito dividido por sus pretensiones, esto es, una persona tendrá una autoestima elevada si percibe que sus logros están a la altura de sus aspiraciones. Así pues, desde esta perspectiva se podría aumentar la autoestima de una persona aumentando sus logros o reduciendo sus aspira-

18. Wolf, Naomi: El mito de la belleza, Barcelona, Emece, 1991, p. 15. 
ciones indistintamente ${ }^{19}$. Ésta es sin duda una definición discutible, puesto que las mismas aspiraciones y logros están fuertemente contaminadas por condicionamientos de tipo cultural y sexista.

Aunque no exenta de problemas, otra posible definición que puede ayudar a comprender qué es y cuáles son los componentes de la autoestima, y de la que se ha derivado un cuestionario bastante utilizado en psicología para medirla, es la que nos proporciona Coopersmith, según la cual la autoestima sería:

«la evaluación que el individuo hace y normalmente mantiene con respecto a sí mismo; expresa una actitud de aprobación o desaprobación e indica la medida en que el individuo se cree capaz, significativo, exitoso y meritorio». ${ }^{20}$

Más recientemente, Branden considera que la autoestima se refiere a "una sensación fundamental de eficacia y un sentido inherente de mérito ${ }^{21}$.

Como señala Marcela Lagarde, tras la Segunda Guerra Mundial surge una preocupación generalizada por la autoestima entre aquellas personas solidarias y conscientes con prisa por reparar los daños ocasionados por la guerra en ellos/as mismos/as y en las personas y comunidades supervivientes ${ }^{22}$.

Esa línea de actuación se extenderá posteriormente a colectivos y comunidades cuyas identidades habían sido menospreciadas o minusvaloradas y que necesitaban modificar la conciencia de sí mismos para luego afrontar y superar las condiciones de desigualdad en las que se hallaban.

En esa corriente se enmarcan las acciones feministas y de género que contribuyen a desarrollar la autoestima de las mujeres para así reparar las heridas y eliminar los sufrimientos generados por la violencia de género y por otras discriminaciones a las que están sometidas ${ }^{23}$.

De hecho, potenciar la autoestima de las mujeres, junto con la mejora de su posición social y de su capacidad para influir en el proceso de transformación de las estructuras sociales, son las claves para lograr el empoderamiento, objetivo clave de estas acciones feministas y de género.

A la vulnerabilidad de la imagen femenina colaboraron muchos de los grandes psicólogos del siglo XX, que optaron por una visión netamente androcéntrica, considerando siempre como norma y referencia básica lo masculino y como secundario lo femenino. Una buena prueba de ello la podemos encontrar en el psicoanálisis. Freud elaboró todo un cuerpo teórico sobre el psiquismo humano considerando el cuerpo y la psique masculina como puntos de referencia indiscutibles. A partir de ahí, a las mujeres se las considerará como «lo

19. HARRÉ, Rom y LAMB, Roger: Diccionario de psicología social y de la personalidad, Barcelona, Paidós, 1992.

20. Coopersmith, S.: The antecedents of Self-Esteem, San Francisco, W. H. Freeman and Co., 1967, p. VII.

21. Branden, Nataniel: Honoring The Self. The Psychology of Confidence and Respect, Nueva York, Bantam Books, 1993, p. 15.

22. IAGARDE, Marcela: Claves feministas para la autoestima de las mujeres, Madrid, Horas y Horas, 2000 .

23. Ibid. 
otro", su cuerpo se definirá a partir de lo que no tienen y desean (el pene) y el sentimiento dominante será el de castración.

«Detrás de esta envidia al miembro viril se vislumbra la hostilidad de la mujer contra el hombre, hostilidad que nunca falta por completo en las relaciones entre los dos sexos y de la cual hallamos claras pruebas en las aspiraciones y producciones literarias de la 'emancipadas'.» ${ }^{24}$

Recogiendo prejuicios muy anteriores a él, y envolviéndolos con la prestigiosa capa de lo supuestamente científico, Freud, como tantos otros, defenderá que cualquier manifestación de independencia, sea personal o intelectual, de las mujeres tiene un significado oculto relacionado con la hostilidad contra los hombres, con un querer ir en contra del orden establecido. Será, por tanto, no sólo algo "contra natura» sino también percibido como peligroso. Quizás por eso la necesidad de doblegarlas, de quebrarlas en su autoimagen y en su autovaloración. Sentirse inseguras con su cuerpo, dudar de su atractivo, considerar partes de él pecaminoso y sucio, es un elemento fundamental en la devaluación de la autoimagen, y ésta es la columna vertebral del yo. Si se quiebra la primera, lo segundo queda también roto. Recordemos dos breves fragmentos de la obra de Freud, que ilustran precisamente el tema del que estamos hablando:

«El pudor es una cualidad por excelencia femenina, cuya intención primaria es encubrir la defectuosidad de sus genitales.» ${ }^{25}$

«La niña, dada su incapacidad de sublimar sus instintos y el predominio de la envidia en su vida mental o anímica, posee escaso sentido de la justicia y débiles intereses sociales. ${ }^{26}$

\section{CONCLUSIÓN}

Estos y tantos otros mensajes parecidos han calado indiscutiblemente en la mente de muchas mujeres que viven su feminidad de una manera angustiosa. Las cifras de chicas jóvenes con problemas de anorexia en el mundo occidental son muy alarmantes. De hecho, en los últimos años la incidencia de trastornos de la conducta alimentaria como la bulimia y la anorexia ha aumentado en España de manera tal que los/as expertos/as califican sus repercusiones sociosanitarias como una «epidemia». Así lo refleja, por ejemplo, Alfonso Chinchilla en un reciente texto según el cual la anorexia nerviosa afectaría a unas 25 personas menores de 25 años por cada 100.000 habitantes, el 90\% de las cuales son mujeres. Según este mismo texto, una de cada 150 mujeres entre 12 y 18 años sufrirá anorexia, mientras que del 1 al 13 por ciento sufrirá bulimia ${ }^{27}$.

Todas ellas tienen un denominador común: odian su cuerpo, lo consideran feo e imperfecto. Algunas de ellas llegarán a la muerte. Todas empezaron su

24. Freud, Sigmund: Obras completas, Madrid, Biblioteca Nueva, 1972, p. 2452.

25. Ibíd., p. 3176.

26. Ibíd., p. 3177.

27. Ginchilla, Alfonso: Trastornos de la conducta alimentaria: anorexia y bulimia nerviosas, obesidad y atracones, Barcelona, Masson, 2002. 
particular calvario optando por una dieta para perder algunos kilos que consideraban sobrantes (en la mayoría de casos no había un sobrepeso real). La presión mediática jugará un papel muy importante. El modelo estético femenino dominante sigue siendo, a pesar de las quejas que empiezan tímidamente a surgir desde diferentes frentes, el de una joven extremadamente delgada, con un aspecto muy frágil, en algunos casos incluso enfermizo. La obsesión por el peso está presente en la mayoría de adolescentes, que lo relacionan con sus expectativas de éxito social. Viven con una enorme presión los cambios corporales relacionados con la maduración y el paso del tiempo. En muchos casos llegan a adultas con una autoestima frágil, quebradiza, y un fuerte sentimiento de frustración, ya que la lucha que tienen declarada está perdida de antemano.

Todo ello no es ni casual ni inocente desde el punto de vista ideológico. Como analiza de manera muy concienzuda Naomi Wolf, el mito de la belleza se convierte en una nueva forma de esclavitud y de freno a la real emancipación femenina, y por contra, en una forma de control masculino sobre las aspiraciones femeninas ${ }^{28}$.

Fragilidad y sumisión siguen siendo pues elementos claves en el estereotipo femenino a día de hoy, a pesar de los grandes cambios vividos y de los avances alcanzados. O quizás debido a ello. El patriarcado, aunque debilitado, no está en absoluto vencido, sigue ejerciendo su tiranía utilizando para ello argumentos más o menos nuevos, y con una enorme carga agresiva que aplica directamente (como es el caso de las diferentes modalidades de violencia de género) o indirectamente, inculcando formas de autoagresión a las jóvenes, cuyas vidas $y$ aspiraciones pueden quedar condicionadas por asumir como prioritario un modelo estético que las convierte en enfermas.

28. Wolf, Naomi: Op. cit. 